Classification

Physics Abstracts

$07.80-61.16 \mathrm{D}-82.80$

\title{
Elemental mapping using an imaging energy filter: image formation and resolution limits
}

\author{
Arthur Berger and Helmut Kohl \\ Institut für Angewandte Physik, Technische Hochschule Darmstadt, Hochschulstraße 6, \\ D-W-6100 Darmstadt, Germany
}

(Received March 27, 1992; accepted May 14, 1992)

\begin{abstract}
Résumé. - Cartographie chimique par filtrage d'énergic: formation de l'image et limite de résolution. L'obtention des cartes chimiques avec un microscope électronique conventionel équipé d'un filtre d'énergie est discutée. On justifie au moyen des calculs numériques la validité de l'approximation dipolaire. Afin de disposer d'un rapport de signal/bruit suffisant, il est indispensable d'utiliser une bande d'énergie finie. C'est pourquoi nous avons calculé la résolution spatiale en fonction de la largeur énergétique sélectionée par le filtre: il nous parait possible de résoudre des structures à l'échelle de 1 nanomètre.
\end{abstract}

\begin{abstract}
The formation of elemental maps in an electron microscope with an imaging energy filter is discussed. The validity of the dipole approximation for the computation of element specific images is demonstrated by explicit comparison with central-field calculations. To obtain a reasonable signal/noise ratio it is vital to use a finite energy window. The attainable resolution is calculated as a function of the width of the energy window. A practical resolution limit of about $1 \mathrm{~nm}$ seems feasible.
\end{abstract}

\section{Introduction.}

The invention of the imaging energy filter thirty years ago by Castaing and Henry [1] has certainly been a milestone in electron microscopy. It permits to form an image or a diffraction pattern using a predetermined energy loss. As this energy loss can be set at will, it can be considered to be a third coordinate in addition to the two lateral coordinates in the image plane. The insertion of an imaging energy filter into the column of an electron microscope thus introduces a new dimension into electron microscopy.

Professor Castaing and his coworkers have subsequently used their new device for numerous studies on fundamental problems in electron microscopy and in solid state physics. They have demonstrated that the contrast in diffraction patterns can be considerably improved by using only the elastically scattered electrons $[2,3,4]$. The same procedure has been used to study the intensity profiles of Kikuchi-lines [5,6]. Furthermore, the preservation of the elastic contrast in plasmon loss images of thickness fringes, bend contours and of stacking faults [7,8], of Fresnel fringes [9] and of Lorentz-images of magnetic domains [10] has been investigated by the Orsay-group. 
Apart from these signals, whose information content stems from the elastic scattering processes, Professor Castaing and his coworkers have shown that precipitates can be imaged using specific plasmon losses $[3,4]$. Similarily voids can be imaged using an appropriate surface plasmon loss [11].

The elemental composition of a specimen can be determined by use of the electrons having lost an energy corresponding to the excitation of an inner-shell characteristic for the element $[12,13]$. Using an imaging energy filter, it is thus possible to produce elemental maps $[14,15,16,17]$. In the following we shall focus on this last aspect.

In recent years, commercial instruments incorporating a Castaing-Henry filter have been used in many laboratories. This has spurred an increasing interest in filtering techniques. A recent review has been given by Reimer [18]. As the Castaing-Henry filter contains an electrostatic mirror, its use is restricted to voltages up to about $80 \mathrm{kV}$. This limitation can be overcome by use of a purely magnetic imaging energy filter. The development of such a filter has started in Orsay [19] and was continued in France [20] and Germany [16,21]. More recently a system has been proposed [22] and constructed [23], which is now commercially available. The results already obtained with this new instrument $[17,24,25]$ demonstrate the large range of applications, where energy filtering leads to results that are not obtainable otherwise.

\section{Theoretical considerations.}

When trying to obtain a faithful representation of the elemental distribution using characteristic energy loss signals, it is essential that the contrast observed is not due to a preservation of the elastic contrast. For any quantitative elemental mapping we must be sure that this indirect effect of elastic scattering processes is negligibly small. This condition ensures that the first order Born approximation can be used.

The calculation of the current density in the image plane is done in two steps. Firstly we determine the wave function of the scattered electrons before the objective lens by using the standard procedures of quantum mechanical scattering theory $[26,27,28,29]$. Secondly we have to calculate the propagation of the electron wave through the lens and into the image plane. This problem is mathematically equivalent to the conventional theory of image formation in light optics or in electron optics for elastically scattered electrons. After some calculations, which are outlined in the appendix, we obtain for the current density $j$ a sum of terms corresponding to the excitation of the object from the ground state $|0\rangle$ to a state $|n\rangle$

$$
\begin{aligned}
& j(\boldsymbol{\rho})=\sum_{n} j_{n 0}(\boldsymbol{\rho}) \\
& =j_{\mathrm{O}} \sum_{n} \frac{k_{\mathrm{f}}}{k_{\mathrm{i}}} \frac{1}{\lambda_{\mathrm{f}}^{2}}\left|\int f_{n 0}(\theta) A(\theta) \exp \{-i \gamma(\boldsymbol{\theta}, \delta E)\} \exp \left\{i k_{\mathrm{f}} \boldsymbol{\theta} \boldsymbol{\rho}\right\} \mathrm{d}^{2} \boldsymbol{\theta}\right|^{2}
\end{aligned}
$$

where $j_{\mathrm{O}}$ is the current density in the object plane, $k_{\mathrm{i}}=2 \pi / \lambda_{\mathrm{i}}$ and $k_{\mathrm{f}}=2 \pi / \lambda_{\mathrm{f}}$ are the wave numbers before and after the scattering process, $f_{n 0}(\theta)$ is the scattering amplitude for the excitation process $|0\rangle \rightarrow|n\rangle$. The function

$$
A(\boldsymbol{\theta})= \begin{cases}1 & \text { for }|\boldsymbol{\theta}| \leq \theta_{0} \\ 0 & \text { otherwise }\end{cases}
$$

describes the objective aperture and 


$$
\gamma(\theta, \delta E)=k_{\mathrm{f}}\left[C_{\mathrm{s}} \frac{\theta^{4}}{4}-\Delta f \frac{\theta^{2}}{2}-C_{\mathrm{c}} \frac{\delta E}{2 E_{0}} \theta^{2}\right]
$$

is the phase shift due to lens aberrations stemming from the spherical aberration $C_{\mathrm{s}}$, the defocus $\Delta f$ and the chromatic aberration $C_{c}$. Here $E_{0}$ and $\delta E$ denote the mean energy of the electron passing through the objective lens and the deviation from it, respectively. We compare this expression with the current density

$$
j_{\mathrm{DF}}(\boldsymbol{\rho})=\frac{j_{\mathrm{O}}}{\lambda^{2}}\left|\int f(\boldsymbol{\theta}) A(\boldsymbol{\theta}) \exp \{-i \gamma(\boldsymbol{\theta}, 0)\} \exp \{i k \rho \boldsymbol{\theta}\} \mathrm{d}^{2} \boldsymbol{\theta}\right|^{2}
$$

in an image obtained in the central dark field mode. The part $j_{n 0}(\rho)$ of the current density in an "inelastic image" is thus obtained by replacing the elastic scattering amplitude $f(\theta)$ by the corresponding amplitude $f_{n 0}(\theta)$ for inelastic scattering (apart from the factor $k_{\mathrm{f}} / k_{\mathrm{i}}$ ). This behaviour can be easily understood because in both cases there is no interference between the unscattered and the scattered waves. The reason for this lack of interference is different though. In central dark field imaging the unscattered beam is intercepted by a small stop in the center of the back focal plane, so the unscattered beam does not contribute to the image. In inelastic imaging there can be no interference because the initial $|0\rangle$ and final $|n\rangle$ states of the object are orthogonal. So even if the unscattered wave passes through the objective aperture and through the energy filter, it can only interfere with scattered waves, which have left the object state unchanged.

For any explicit computation for a given object we first have to determine the transition amplitudes $f_{n 0}(\theta)$. As we are interested in the use of inner-shell losses for elemental mapping, we suppose that the atoms are independant from each other. This assumption is already implicitly made when one states that a particular atom has been excited. Furthermore, we use free-atom matrix elements, thus neglecting the fine structure effects, which are due to the modification of the final atomic states in a solid. As one uses rather large energy windows, these fine structure effects are averaged out.

The local chemical composition of the specimen is described by the particle densities $n_{\nu}(\rho)$ for each element $\nu=1,2, \ldots$. We now consider a range of atomic transitions, which are assumed to be characteristic for a given element $\nu$. To determine the current density $j_{\mathrm{T}}(\rho)$ within a given energy window $\Delta E \pm \delta E / 2$, we have to sum over all atoms $\ell$ of type $\nu$ at positions $\rho_{\ell}$

$$
j_{\mathrm{T}}(\boldsymbol{\rho})=\sum_{\ell} j_{\mathrm{A}}\left(\boldsymbol{\rho}-\boldsymbol{\rho}_{\ell}\right),
$$

where $j_{\mathrm{A}}(\rho)$ is the current density due to a single atom at the origin.

We note that the relation (5) does not hold true for "elastic" dark field imaging. In this case we must consider the interference between waves scattered by different atoms. For inelastic scattering any two states describing the atom $\ell$ and $\ell^{\prime}$ are orthogonal and therefore there is no interference between the corresponding partial waves. Equation (5) states that the total current density is a sum of terms due to single atoms. Due to this linear relation, a Fourier transform of equation (5) yields the standard form of any linear imaging theory

$$
J(\Omega)=N_{\nu}(\Omega) J_{\mathbf{A}}(\Omega)
$$

where

$$
J(\Omega)=\int j_{\mathrm{T}}(\rho) \exp \left\{i k_{\mathrm{f}} \Omega \rho\right\} \mathrm{d}^{2} \rho
$$


and

$$
J_{\mathrm{A}}(\boldsymbol{\Omega})=\int j_{\mathrm{A}}(\boldsymbol{\rho}) \exp \left\{i k_{\mathrm{f}} \boldsymbol{\Omega} \rho\right\} \mathrm{d}^{2} \rho
$$

are the Fourier transformed current densities and

$$
N_{\nu}(\boldsymbol{\Omega})=\int n_{\nu}(\rho) \exp \left\{i k_{\mathrm{f}} \boldsymbol{\Omega} \rho\right\} \mathrm{d}^{2} \rho=\sum_{j} \exp \left\{i k_{\mathrm{f}} \boldsymbol{\Omega} \rho_{j}\right\}
$$

is the Fourier transform of the particle density $n_{\nu}(\rho)$ of the element $\nu$ in the object. For a given specimen $n_{\nu}(\rho)$ and thus $N_{\nu}(\Omega)$ is known. To determine $J(\Omega)$ we have to calculate $J_{\mathrm{A}}(\Omega)$, which we shall write as

$$
J_{\mathrm{A}}(\Omega)=j_{\mathrm{O}} \sigma H(\Omega)
$$

where $\sigma$ is the inelastic cross-section for the chosen energy window and the objective aperture. The function $H(\Omega)$ is a transfer function, which is dependent on the instrumental parameters and on the transition under consideration.

\section{The dipole approximation.}

For determining the transfer function, or equivalently, the current density $j_{\mathrm{A}}(\rho)$ we must know the inelastic scattering amplitudes $f_{n 0}(\theta)$ for the considered transitions. Within the frame of the first-order Born approximation $f_{n 0}(\theta)=f_{n 0}(\mathbf{K})$ is given by the matrix element

$$
f_{n 0}(\mathbf{K})=-\frac{m_{0}}{2 \pi \hbar^{2}}\left\langle\mathbf{k}_{\mathbf{f}} n|\mathbf{V}| \mathbf{k}_{i} 0\right\rangle=-\frac{2}{K^{2} a_{\mathbf{H}}}\langle n|\exp \{i \mathbf{K r}\}| 0\rangle
$$

for the excitation of an electron from its initial state $|0\rangle$ to a final state $|n\rangle$. Here $\mathbf{K}=\mathbf{k}_{\mathbf{i}}-\mathbf{k}_{\mathbf{f}}$ denotes the scattering vector and $a_{\mathrm{H}}=0.529 \AA$ is Bohrs radius. For small scattering angles and thus small scattering vectors, the exponential function can be expanded

$$
\exp \{i \mathbf{K r}\} \approx 1+i \mathbf{K r} \text {. }
$$

Considering the orthogonality relation

$$
\langle n \mid 0\rangle=0
$$

this procedure yields

$$
f_{n 0}(\mathbf{K})=\frac{-2 i}{K^{2} a_{\mathrm{H}}}\langle n|\mathbf{K} \mathbf{r}| 0\rangle .
$$

In this dipole approximation the scattering amplitude is given by the dipole matrix element $\langle n|\mathbf{r}| 0\rangle$ multiplied by $\frac{-2 i K}{K^{2} a_{H}}$. Therefore the specific transition enters only via a factor describing the strength of the transition, whereas the dependence on $\mathbf{K}$ is completely determined [30].

To determine the range of validity of the dipole approximation we have calculated the current density for the inner-shell excitation of a single atom, using a central-field model for the atomic potential [31] and assuming an ideal lens $(\gamma=0)$. The results for the K-excitation of oxygen are shown in figure 1. As has been shown previously [30], there is a ring of high intensity around the atom. We can see that for an objective aperture angle of $10 \mathrm{mrad}$ the "exact" calculation yields only minor corrections to the dipole approximation. To check the accuracy of the dipole 


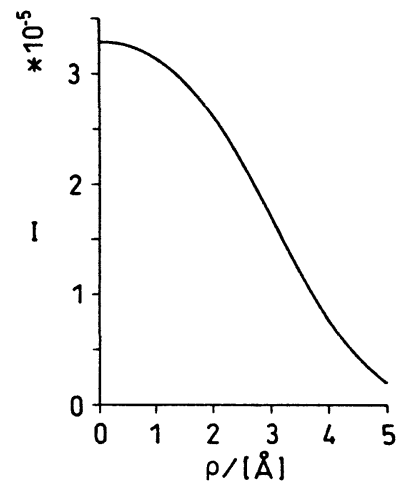

a)

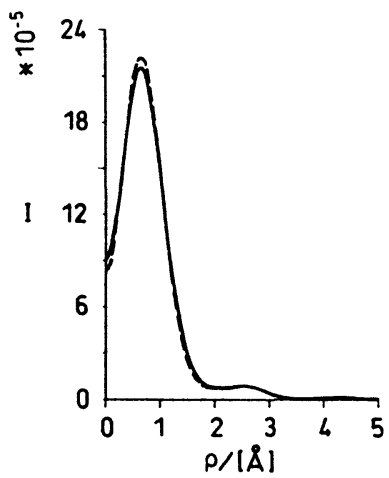

c)

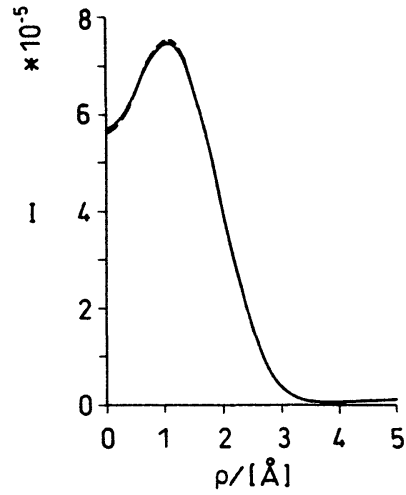

b)

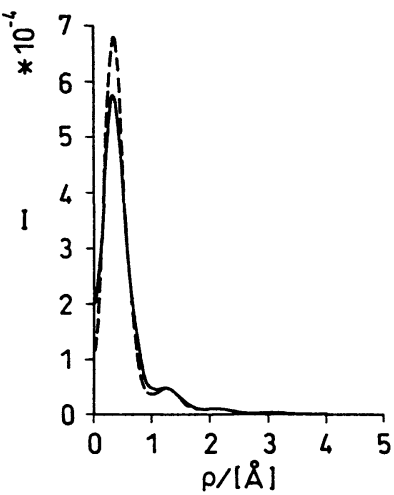

d)

Fig. 1. - Intensity distribution in the image of an oxygen atom when using only K-loss electrons. An electron energy of $120 \mathrm{keV}$ and an ideal lens $(\gamma=0)$ are assumed. The results using the matrix elements from a central field model (full curve) and those obtained by the dipole approximation (dashed curve) are shown for different objective apertures of (a) $5 \mathrm{mrad}$ (b) $10 \mathrm{mrad}$ (c) $20 \mathrm{mrad}$ and (d) $40 \mathrm{mrad}$.

approximation further, we have performed a series of calculations variing the edge type and the energy loss. Some results are displayed in figures 2 and 3. In figure 2 we compare the single-atom images for the $\mathrm{O}-\mathrm{K}$, the $\mathrm{V}-\mathrm{L}_{23}$ and the $\mathrm{Sn}-\mathrm{M}_{45}$ excitations with the corresponding dipole approximation. As the energy losses are equal, the dipole approximation predicts the same shape for each of these three excitations. To be able to discern any differences, we have assumed an extremely large objective aperture angle of $40 \mathrm{mrad}$. From the curves we can see that even though there are some differences, these are relatively small. For any realistic aperture angle (typically about $10 \mathrm{mrad}$ ) the dipole approximation yields reliable results irrespective of the edge type. To investigate the dependence on the energy loss, we have performed calculations for the $\mathrm{Li}-\mathrm{K}$ and for the $\mathrm{Al}-\mathrm{K}$ edge. We find that for low energy losses and an aperture angle of $10 \mathrm{mrad}$, the deviations from the dipole approximation are somewhat larger, whereas for high energy losses, the dipole approximation is valid even for aperture angles of $40 \mathrm{mrad}$. We can thus safely state that for realistic imaging parameters the use of the dipole approximation is well justified. 


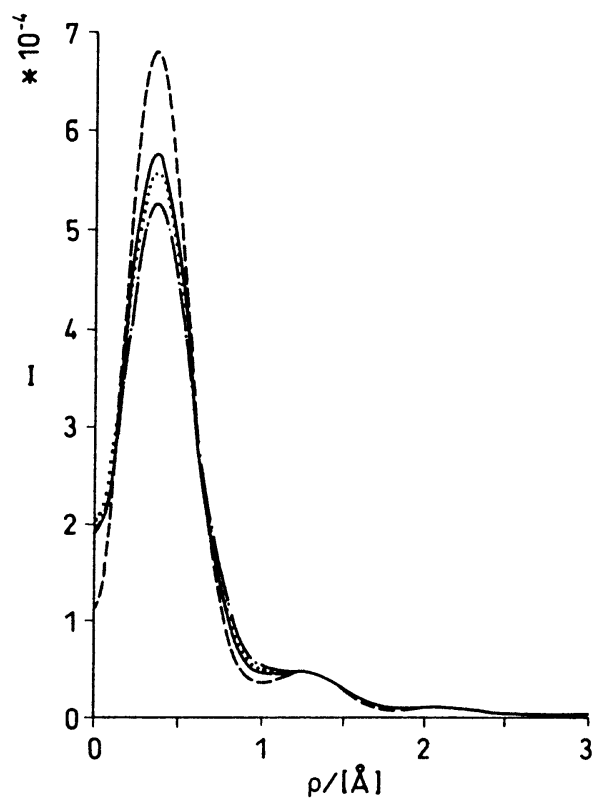

Fig. 2. - Intensity distribution in the image of a single atom in the case of oxygen K-loss (full curve), vanadium $L_{23}$-loss (dotted curve), and tin $M_{45}$-loss (dash-dotted curve) for a given energy loss of $\Delta E=540 \mathrm{eV}$, an electron energy of $120 \mathrm{keV}$ and an objective aperture angle of $40 \mathrm{mrad}$. The central field model and the dipole approximation (dashed curve) have been employed.

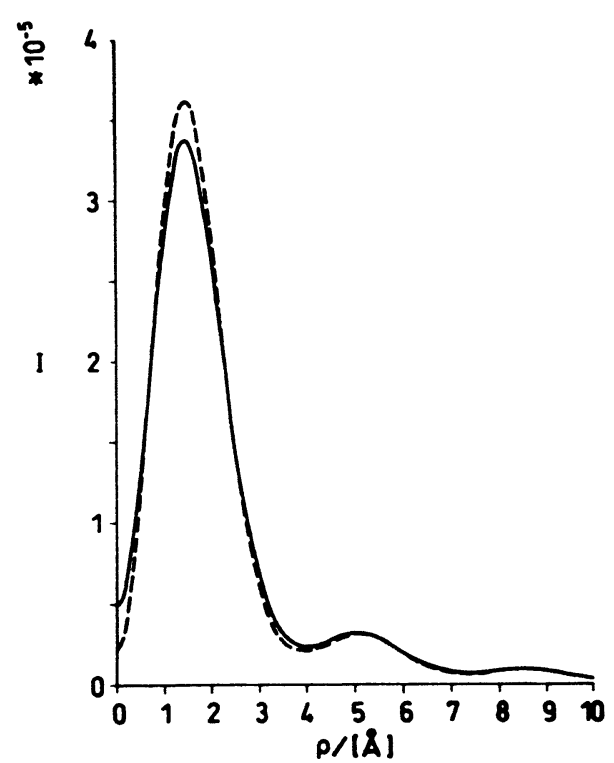

a)

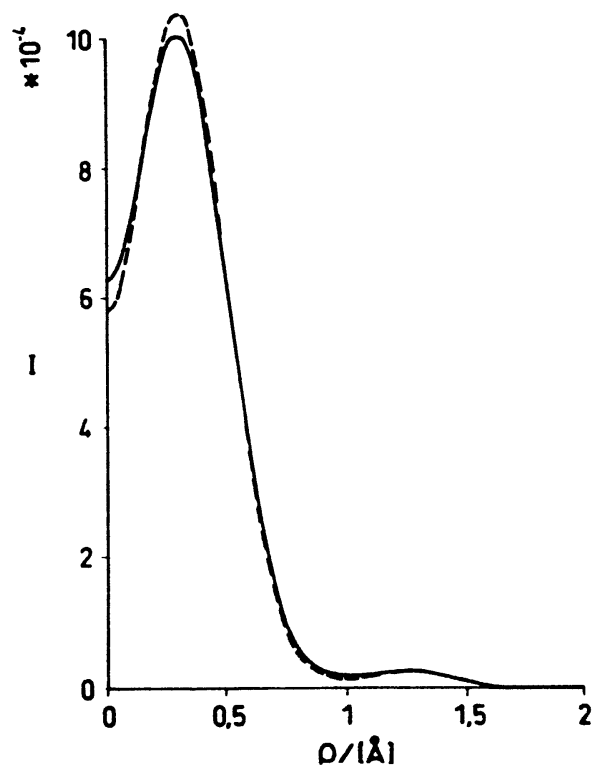

b)

Fig. 3. - Results of central field calculations (full curves) and the dipole approximation (dashed curves) for (a) $\mathrm{Li}-\mathrm{K}$ (with $\theta_{0}=10 \mathrm{mrad}$ and $\Delta E=65 \mathrm{eV}$ ) and (b) $\mathrm{Al}-\mathrm{K}$ (with $\theta_{0}=40 \mathrm{mrad}$ and $\Delta E=1580 \mathrm{eV}$ ) loss images. 


\section{The influence of lens aberrations.}

The aberrations of electron lenses play an important role in the image formation process. To assess their influence for elemental maps, we have calculated the images for an $\mathrm{O}-\mathrm{K}$ excitation with and without taking the lens aberrations into account. For comparison we have drawn the equivalent curves for the image of a single heavy atom in the dark field mode. The differences between figures $4 \mathrm{a}$ and $\mathbf{4 b}$ are due to the inelastic scattering process. As the extension of the atomic shells is much smaller than the resolution limit of current instruments, the intensity distribution in the (elastic)

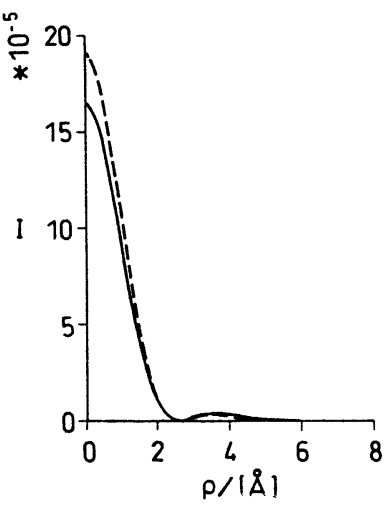

a)

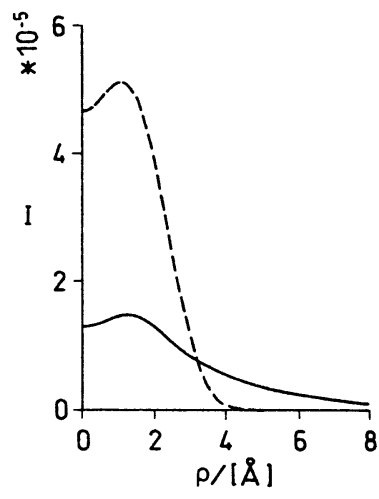

b)

Fig. 4. - Intensity distribution in the image of an atom employing an accelerating voltage of $120 \mathrm{keV}$ and an objective aperture angle of $\theta_{0}=8.5 \mathrm{mrad}$ for (a) the "elastic" dark-field mode; (b) $540 \mathrm{eV}$-loss electrons (corresponding to the oxygen K-loss). The dashed curves show the results for an ideal lens $(\gamma=0)$, whereas the full curves have been calculated for the aberration constants of the Zeiss EM 912 Omega instrument $\left(C_{\mathrm{s}}=2.7 \mathrm{~mm}, C_{\mathrm{c}}=2.7 \mathrm{~mm}\right)$. In (a) we have assumed the "optimal" defocus of $\Delta f=0.82 \sqrt{C_{\mathrm{s}} \lambda}=$ $780 \AA$, whereas in (b) we have assumed an energy window $\delta E=20 \mathrm{eV}$ and an optimized defocus of $\Delta f=$ $0.1 \sqrt{C_{\mathrm{s}} \lambda}=95 \AA$.

dark field image is an Airy disc for an ideal lens (dashed curve). This disc is only sightly changed by the spherical aberration of the objective lens (full curve). However, the intensity distribution in the inelastic image is somewhat broader than for a point scatterer. This is easily explained by the fact that an incident electron passing an atom at some distance still interacts with the bound electrons and can therefore cause transitions. To obtain an elemental map, we have to use a finite energy window, whose size we have assumed to be $20 \mathrm{eV}$. Then the intensity distribution is considerably broader due to the chromatic aberration. The important parameter is the normalized focal spread $\kappa$, which is given by the ratio of the focal spread $C_{\mathrm{c}} \frac{\delta E}{E}$ to the Scherzer defocus $\sqrt{C_{\mathrm{s}} \lambda}$ [32]. In our case this parameter is about 5 , meaning that the focal spread is much larger than the Scherzer defocus. In figure 5 we have drawn the transfer functions corresponding to the image intensities in figure 4. The decrease in resolution for large energy windows manifests itself in a rapid decrease of the transfer function for large spatial frequencies in figure $5 \mathrm{~b}$. For typical energy windows the attainable resolution is thus limited by the chromatic aberration. To illustrate this behavior we have calculated the resolution limit $d$ as defined by the diameter of the disc containing $59 \%$ of the total intensity as a function of the normalized focal spread $\kappa$. For a given focal spread we have computed the defocus and the objective aperture angle giving the smallest value of $d$. The results 


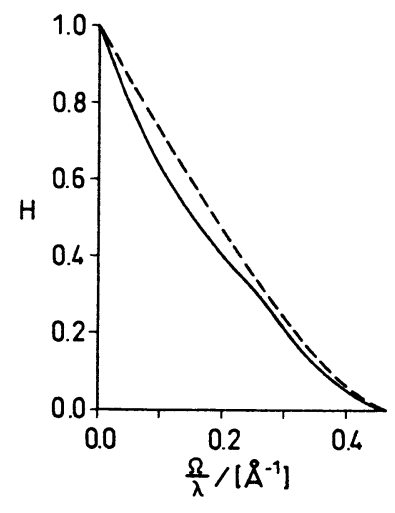

a)

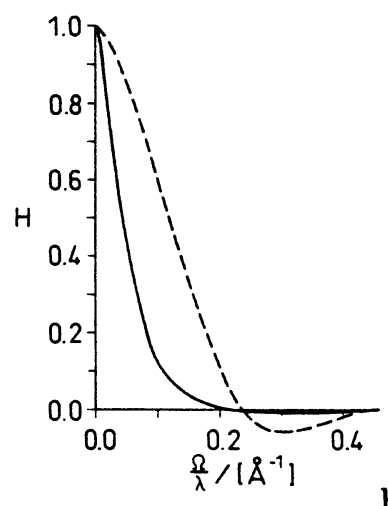

Fig. 5. - Normalized Fourier-transforms of the curves shown in figure 4 yielding (a) the modulation transfer function and (b) the transfer function for inelastic imaging.

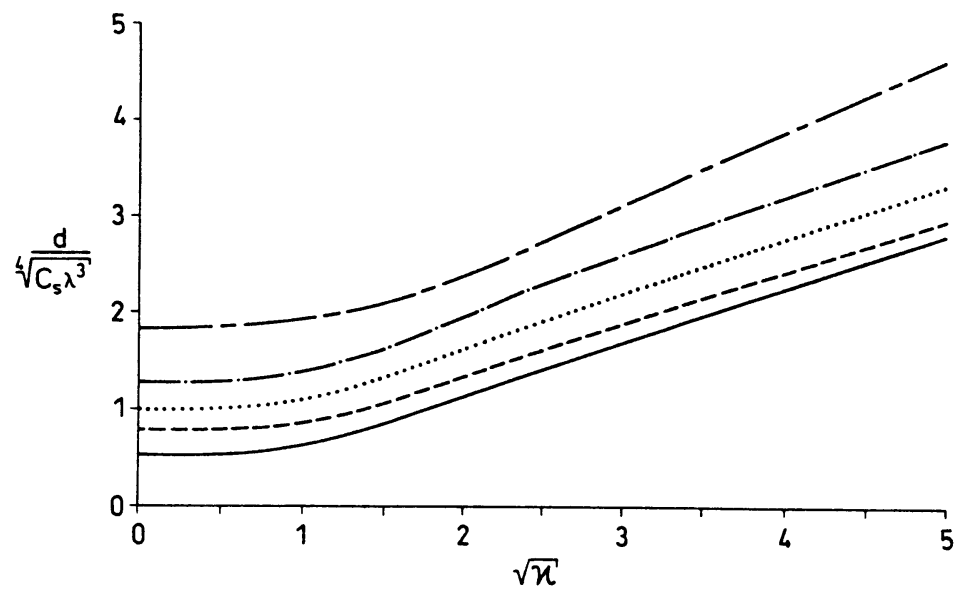

a)
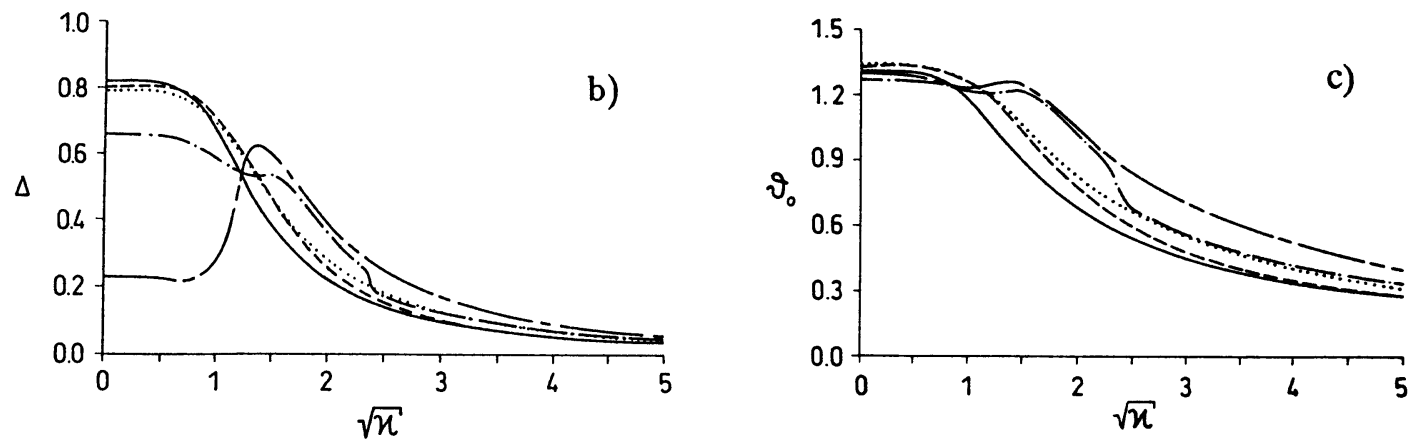

Fig. 6. - (a) Normalized resolution limit $d / \sqrt[4]{C_{\mathrm{s}} \lambda^{3}}$ as a function of the energy window expressed by the normalized focal spread $\kappa=\frac{C_{\mathrm{c}}}{\sqrt{C_{\mathrm{s}} \lambda}} \frac{\delta E}{E_{\mathrm{o}}}$ for normalized energy loss angles $\vartheta_{\mathrm{E}}=\frac{\Delta E}{E_{\mathrm{o}}} \frac{m_{0} c^{2}+E_{o}}{2 m_{0} c^{2}+E_{o}} / \sqrt[4]{\frac{\lambda}{C_{\mathrm{s}}}}$ of $\infty(-), 0.5(-\cdots-), 0.2(\ldots \ldots \ldots), 0.1(-\cdots \cdots)$, and $0.05(---)$. (b) normalized defocus $\Delta=\Delta f / \sqrt{C_{\mathrm{s}} \lambda}$ and (c) the normalized optimal objective aperture angle $\vartheta_{0}=\theta_{0} / \sqrt[4]{\frac{\lambda}{C_{\mathrm{s}}}}$. 
for several energy losses are presented in figure 6. Looking at figure 6a we find that up to $\kappa=1$ the chromatic aberration hardly influences the attainable resolution. For larger energy windows the resolution decreases with an asymptote proportional to $\sqrt{\kappa}$. Figures $6 \mathrm{~b}$ and $6 \mathrm{c}$ show the objective aperture and the defocus value necessary to obtain the optimal resolution. The sudden humps are due to the fact that the resolution limit as a function of aperture angle $\vartheta_{0}$ and defocus $\Delta$ has two local minima, whose relative height changes with $\kappa$. At several critical values of $\kappa$ bifurcations occur. As $\kappa$ denotes the focal spread in Scherzer units, the resolution for large energy windows is not sensitive to the defocus. For these large $\kappa$ values the optimal aperture angle decreases, thus balancing the effect of the chromatic aberration and the diffraction disc.

To demonstrate the influence of the accelerating voltage and the aberration constants on the resolution attainable for given energy losses and energy windows we have compared the theoretical resolution limit of a Zeiss EM $912 \Omega$ with the values for a (fictitious) filter microscope with the Philips ultra twin lens at $120 \mathrm{kV}$ and at $200 \mathrm{kV}$, respectively. The instrumental parameters are shown in table I. In table II we have listed the resolution limits for a low $(\Delta E=200 \mathrm{eV})$ and a

Table I. - Instrumental parameters used for the calculation of theoretical resolution limits for elemental maps.

\begin{tabular}{|l|c|c|c|}
\hline & EM 912 $\Omega$ & CM 12 Ultra Twin & CM 20 Ultra Twin \\
\hline$E_{0} /[\mathrm{keV}]$ & 120 & 120 & 200 \\
\hline$C_{\mathrm{S} /[\mathrm{mm}]}$ & 2.7 & 0.5 & 0.5 \\
\hline$C_{\mathrm{C} /[\mathrm{mm}]}$ & 2.7 & 1.0 & 1.0 \\
\hline \hline$\left.\sqrt[4]{C_{\mathrm{S}} \lambda^{3} /[\AA]}\right]$ & 5.6 & 3.7 & 3.0 \\
\hline$\sqrt{C_{\mathrm{S}} \lambda /[\AA]}$ & 951 & 409 & 354 \\
\hline$\sqrt[4]{\frac{\lambda}{C_{\mathrm{S}}} /[\mathrm{mrad}]}$ & 5.9 & 9.1 & 8.4 \\
\hline$\vartheta_{\mathrm{E}}(E=200 \mathrm{eV})$ & 0.16 & 0.1 & 0.07 \\
\hline
\end{tabular}

Table II. - Theoretical resolution limits for elemental mapping in a filter microscope. We have compared the results for a Zeiss EM $912 \Omega$ with those of a (hypothetical) filter microscope employing an objective lens with the aberration constants of the Philips Ultra Twin lens. For the calculation we have assumed a high $(\Delta E \rightarrow \infty)$ and a low $(\Delta E=200 \mathrm{eV})$ energy loss and energy windows $\delta E$ of $10 \mathrm{eV}, 20 \mathrm{eV}$ and $40 \mathrm{eV}$.

\begin{tabular}{|c|c|c|c|c|}
\hline$\Delta E /[\mathrm{eV}]$ & $\delta E /[\mathrm{eV}]$ & \multicolumn{3}{|c|}{$d /[\AA]$} \\
\cline { 3 - 5 } & & EM 912 $\Omega$ & CM 12 Ultra Twin & CM 20 Ultra Twin \\
\hline$\infty$ & $10 \mathrm{eV}$ & 4.9 & 3.0 & 2.1 \\
\hline$\infty$ & $20 \mathrm{eV}$ & 6.9 & 4.2 & 2.8 \\
\hline$\infty$ & $40 \mathrm{eV}$ & 9.9 & 5.9 & 4.0 \\
\hline $200 \mathrm{eV}$ & $10 \mathrm{eV}$ & 8.1 & 5.9 & 5.0 \\
\hline $200 \mathrm{eV}$ & $20 \mathrm{eV}$ & 10.3 & 7.3 & 5.7 \\
\hline $200 \mathrm{eV}$ & $40 \mathrm{eV}$ & 13.3 & 9.3 & 7.2 \\
\hline
\end{tabular}


high $(\Delta E \rightarrow \infty)$ energy loss, assuming energy windows of $\delta E=10 \mathrm{eV}, 20 \mathrm{eV}$ and $40 \mathrm{eV}$. The values clearly demonstrate the improvement in resolution with decreasing aberration constants and increasing accelerating voltage.

We stress the fact that we have optimized the resolution for a given energy window. In practise, however, the attainable resolution is limited by the signal/noise ratio (SNR) rather than by the instrumental parameters. Therefore, we should optimize the SNR rather than the resolution. Preliminary calculations indicate that it is advantageous to use larger objective apertures and smaller values of $\kappa$ to obtain a given resolution. We are currently undertaking a systematic study of this problem.

\section{Conclusion.}

The formation of elemental maps by use of inelastically scattered electrons has become a widely used technique, particularily useful for light elements. We have discussed the basic assumptions and approximations underlying the theory of image formation for inelastically scattered electrons in an energy filtering transmission electron microscope. In particular we have demonstrated the applicability of the dipole approximation. The attainable resolution for typical width of the energy windows is limited by the effect of the chromatic aberration of the objective lens. Nevertheless a practical resolution limit of about $1 \mathrm{~nm}$ seems to be achievable. This value is almost the same as that obtained in a scanning transmission electron microscope $[33,34]$. For both instruments the ultimate limit is imposed by signal/noise considerations [35].

\section{Acknowledgements.}

We would like to thank Dr. D. Krahl (Fritz-Haber-Institut,Berlin) for valuable discussions and Prof. H. Rose (TH Darmstadt) for critical reading of the manuscript. One of us (HK) wishes to thank Drs. C. Colliex, C. Mory, M. Achèche and M. Tencé for fruitful discussions during a oneyear stay in Orsay. Financial support by the Deutsche Forschungsgemeinschaft (Ko 885/3-1 and 3-2) is gratefully acknowledged.

\section{Appendix I}

In this section we shall outline the derivation of equation (1). As is well known for images with elastically scattered electrons, the essential part of the imaging system is the objective lens and the aperture. The geometry is shown in figure 7.

To calculate the current density in the image plane, we proceed from left to right. The incident electron with momentum $\hbar \mathbf{k}$ is described by a plane wave exp $\{i k z\}$. It is scattered by the object located at the plane $z=z_{\mathrm{O}}$. For simplicity we set $z_{\mathrm{O}}=0$. The emanating partial waves are focussed by the objective lens, situated in the plane $z_{\mathrm{L}}$. This effect of the lens with a focal length $f$ can be described by a transmission function [36]

$$
T_{\mathrm{L}}\left(\rho_{\mathrm{L}}\right)=\exp \left\{-i \frac{k}{2 f} \rho_{\mathrm{L}}^{2}\right\}
$$

acting upon the wave function $\psi_{\mathrm{L}}\left(\rho_{\mathrm{L}}\right)$ in the plane immediately before the lens. Using Huygens principle in the Fresnel-approximation we derive the wave function in the back-focal plane $(z=$ $\left.z_{\mathrm{A}}\right)$. At this plane the influence of the objective aperture and the axial aberrations can be described 


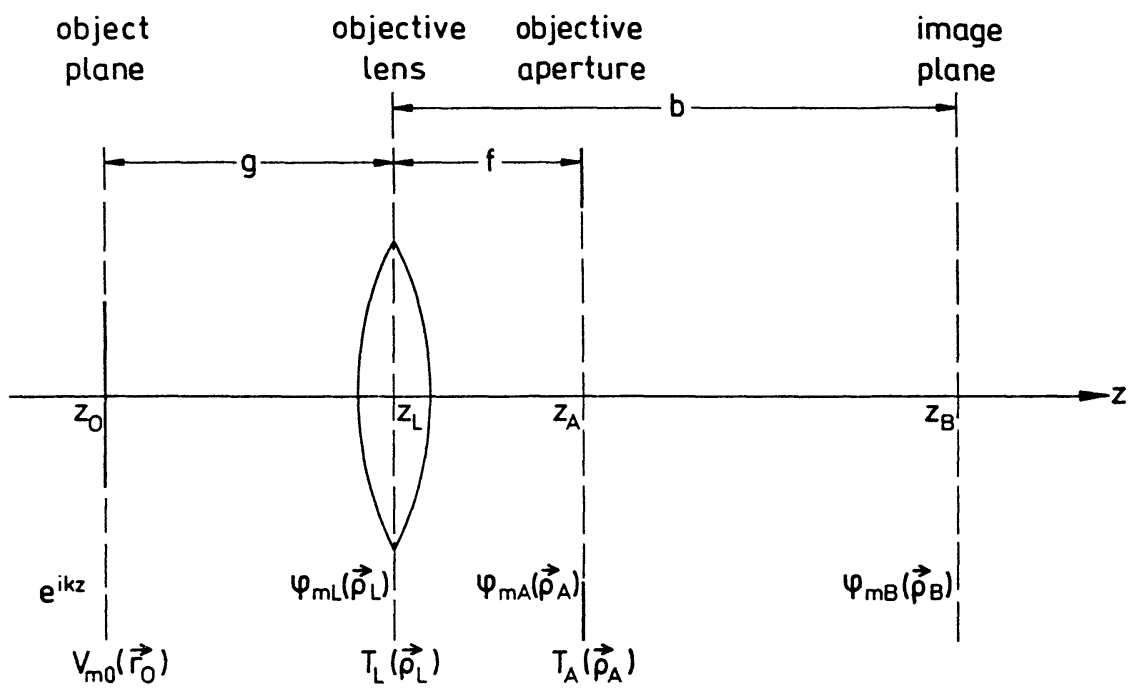

Fig. 7. - Schematic diagram depicting the image formation using the electrons, which leave the object in an excited state $|m\rangle$.

by the transmission function

$$
T_{\mathrm{A}}(\boldsymbol{\theta})=A(\boldsymbol{\theta}) \exp \{-i \gamma(\boldsymbol{\theta}, \delta E)\}
$$

where $\theta=\rho_{\mathrm{A}} / f$ corresponds to the scattering vector.The aperture function

$$
A(\boldsymbol{\theta})= \begin{cases}1 & \text { for }|\boldsymbol{\theta}| \leq \theta_{0} \\ 0 & \text { otherwise }\end{cases}
$$

considers the influence of the beam limiting diaphragm and

$$
\gamma(\theta, \delta E)=k\left\{C_{\mathrm{s}} \frac{\theta^{4}}{4}-\Delta f \frac{\theta^{2}}{2}-C_{\mathrm{c}} \frac{\delta E}{2 E_{0}} \theta^{2}\right\}
$$

is the phase shift due to defocus and lens aberrations. Again using Huygens principle in the Fresnel approximation we shall eventually obtain the wave function at the image plane $z=z_{\mathrm{B}}$. This function yields the final current density.

Following the passage of the electron through the microscope we now consider the inelastic scattering of a plane wave $\exp \left\{i k_{\mathrm{i}} z\right\}$ by the specimen $[26,27,28,29]$. The Hamilton operator $\mathrm{H}$ is given by the sum

$$
\mathrm{H}=\mathrm{H}^{0}+\mathrm{V}=\mathrm{H}_{\mathrm{E}}+\mathrm{H}_{\mathrm{O}}+\mathrm{V}
$$

where $\mathrm{H}_{\mathrm{E}}$ and $\mathrm{H}_{\mathrm{O}}$ are the Hamilton operator of the incident electron and the object, respectively. The operator $\mathrm{V}$ describes the interaction between the incident electron and the electrons of the object. The solution can be expanded into a sum of product states

$$
\psi(\mathbf{r}, \mathbf{R})=\exp \left\{i k_{\mathbf{i}} z\right\} \Phi_{0}(\mathbf{R})+\sum_{n} \varphi_{n}(\mathbf{r}) \Phi_{n}(\mathbf{R})
$$


where the functions $\Phi_{n}(\mathbf{R})$ are eigenstates of the operator $H_{O}$ :

$$
H_{\mathrm{O}} \Phi_{n}(\mathbf{R})=E_{n} \Phi_{n}(\mathbf{R}) .
$$

The expansion coefficients are the functions $\varphi_{n}(\mathbf{r})$ of the position $\mathbf{r}$ of the incident electron. The vector $\mathbf{R}=\left(\mathbf{r}_{1}, \mathbf{r}_{2}, \mathbf{r}_{3}, \ldots \ldots \ldots, \mathbf{r}_{N}\right)$ is used as a short hand notation for the positions of the $\mathbf{N}$ electrons in the specimen. Since the center of each atom within the object has been fixed, phonon excitations are disregarded.

The initial state of the system given by

$$
\psi_{\mathrm{i}}(\mathbf{r}, \mathbf{R})=\exp \left\{i k_{\mathrm{i}} z\right\} \Phi_{0}(\mathbf{R})
$$

has an energy of $E=E_{0}+\frac{\hbar^{2} k_{\mathrm{i}}^{2}}{2 m_{0}}$. The interaction operator has the form

$$
V(\mathbf{r}, \mathbf{R})=\frac{e^{2}}{4 \pi \epsilon_{0}} \sum_{j} \frac{1}{\left|\mathbf{r}-\mathbf{r}_{j}\right|}
$$

To determine the functions $\varphi_{n}(\mathbf{r})$, we insert the expression (20) into the Schrödinger equation

$$
\left(\mathrm{H}_{\mathrm{E}}+\mathrm{H}_{\mathrm{O}}+\mathrm{V}\right) \psi=E \psi .
$$

Multiplying by $\Phi_{m}^{*}(\mathbf{R})$ from the left and integrating over the object coordiantes we obtain the equation

$$
\left\{\mathrm{H}_{\mathrm{E}}-\frac{\hbar^{2} k_{m}^{2}}{2 m_{0}}\right\} \varphi_{m}(\mathbf{r})+\exp \left\{i k_{\mathrm{i}} z\right\} V_{m 0}(\mathbf{r})+\sum_{n} \varphi_{n}(\mathbf{r}) V_{m n}(\mathbf{r})=0
$$

where

and

$$
E-E_{m}=\frac{\hbar^{2} k_{m}^{2}}{2 m_{0}}
$$

Using

$$
V_{m n}(\mathbf{r})=\int \Phi_{m}^{*}(\mathbf{R}) V(\mathbf{r}, \mathbf{R}) \Phi_{n}(\mathbf{R}) \mathbf{d}^{3 N} \mathbf{R}
$$

$$
\mathrm{H}_{\mathrm{E}}=-\frac{\hbar^{2}}{2 m_{0}} \Delta
$$

we can rearrange (25)

$$
\left(\Delta+k_{m}^{2}\right) \varphi_{m}(\mathbf{r})=\frac{2 m_{0}}{\hbar^{2}}\left\{\exp \left\{i k_{\mathrm{i}} z\right\} V_{m 0}(\mathbf{r})+\sum_{n} \varphi_{n}(\mathbf{r}) V_{m n}(\mathbf{r})\right\},
$$

thus obtaining a system of coupled differential equations. If the scattering potential is sufficiently weak, the resulting functions $\varphi_{n}$ are small. Then we can use the first order Born approximation, thus neglecting the sum over $n$ on the right hand side, whose terms are quadratically small. The remaining equation

$$
\left(\Delta+k_{m}^{2}\right) \varphi_{m}(\mathbf{r}) \approx \frac{2 m_{0}}{\hbar^{2}} \exp \left\{i k_{\mathrm{i}} z\right\} V_{m 0}(\mathbf{r})
$$


is readily solved by use of the Green-function

$$
G_{m}\left(\mathbf{r}-\mathbf{r}^{\prime}\right)=\frac{-1}{4 \pi} \frac{\exp \left\{i k_{m}\left|\mathbf{r}-\mathbf{r}^{\prime}\right|\right\}}{\left|\mathbf{r}-\mathbf{r}^{\prime}\right|}
$$

yielding

$$
\varphi_{m}(\mathbf{r})=-\frac{m_{0}}{2 \pi \hbar^{2}} \int \frac{\exp \left\{i k_{m}\left|\mathbf{r}-\mathbf{r}^{\prime}\right|\right\}}{\left|\mathbf{r}-\mathbf{r}^{\prime}\right|} \exp \left\{i k_{\mathrm{i}} z^{\prime}\right\} V_{m 0}\left(\mathbf{r}^{\prime}\right) \mathbf{d}^{3} \mathbf{r}^{\prime}
$$

In standard scattering theory one is interested in $\varphi_{m}(\mathbf{r})$ at extremely large distances from the scattering object $(|\mathbf{r}| \rightarrow \infty)$. One then uses the Fraunhofer-approximation [26, 27, 28, 29]. Here, however, we need to know $\varphi_{m}$ in the plane $z_{\mathrm{L}}$ just before the objective lens. Therefore we must apply the Fresnel approximation for the spherical wave [36. Using the expansion

$$
\begin{aligned}
\left|\mathbf{r}_{\mathrm{L}}-\mathbf{r}^{\prime}\right| & =\sqrt{\left(z_{\mathrm{L}}-z^{\prime}\right)^{2}+\left(\rho_{\mathrm{L}}-\rho_{\mathrm{O}}\right)^{2}}=g \sqrt{1-\frac{2 z^{\prime}}{g}+\frac{z^{\prime 2}}{g^{2}}+\frac{\left(\rho_{\mathrm{L}}-\rho_{\mathrm{O}}\right)^{2}}{g^{2}}} \\
& \approx g-z^{\prime}+\frac{1}{2 g}\left(\rho_{\mathrm{L}}-\rho_{\mathrm{O}}\right)^{2}
\end{aligned}
$$

for the exponent of the Green function and the approximation

$$
\left|\mathbf{r}_{\mathrm{L}}-\mathbf{r}^{\prime}\right| \approx g=z_{\mathrm{L}}-z_{\mathrm{O}}
$$

for its nominator we obtain

$$
\frac{\exp \left\{i k_{m}\left|\mathbf{r}_{\mathrm{L}}-\mathbf{r}^{\prime}\right|\right\}}{\left|\mathbf{r}_{\mathrm{L}}-\mathbf{r}^{\prime}\right|} \approx \frac{\exp \left\{i k_{m}\left(g-z^{\prime}\right)\right\}}{g} \exp \left\{i \frac{k_{m}}{2 g}\left(\rho_{\mathrm{L}}-\rho_{O}\right)^{2}\right\} .
$$

In the plane $z=z_{\mathrm{L}}$ immediately before the lens, which we assume to be infinitely thin, the partial wave function $\varphi_{m}$ is given by

$$
\begin{gathered}
\varphi_{m}\left(\rho_{\mathrm{L}}, z_{\mathrm{L}}\right)=\varphi_{m \mathrm{~L}}\left(\rho_{\mathrm{L}}\right)=-\frac{m_{0}}{2 \pi \hbar^{2}} \frac{\exp \left\{i k_{m} g\right\}}{g} \\
\cdot \int \exp \left\{i\left(k_{\mathrm{i}}-k_{m}\right) z^{\prime}\right\} V_{m 0}\left(\rho_{\mathrm{O}}, z^{\prime}\right) \exp \left\{i \frac{k_{m}}{2 g}\left(\rho_{\mathrm{L}}-\rho_{\mathrm{O}}\right)^{2}\right\} \mathrm{d}^{2} \rho_{\mathrm{O}} \mathrm{d} z^{\prime} .
\end{gathered}
$$

To determine the wave function in the back focal plane, we have to multiply $\varphi_{m L}$ with the transmission function $T_{\mathrm{L}}\left(\rho_{\mathrm{L}}\right)$ describing the action of the lens and calculate the Sommerfeld integral in the Fresnel-approximation [36].

$$
\begin{aligned}
\varphi_{m \mathrm{~A}}\left(\rho_{\mathrm{A}}\right) & =\frac{\exp \left\{i k_{m} f\right\}}{i \lambda_{m} f} \int \varphi_{m \mathrm{~L}}\left(\rho_{\mathrm{L}}\right) T_{\mathrm{L}}\left(\rho_{\mathrm{L}}\right) \exp \left\{i \frac{k_{m}}{2 f}\left(\rho_{\mathrm{A}}-\rho_{\mathrm{L}}\right)^{2}\right\} \mathrm{d}^{2} \rho_{\mathrm{L}} \\
& =-\frac{m_{0}}{2 \pi \hbar^{2}} \frac{\exp \left\{i k_{m}(f+g)\right\}}{i \lambda_{m} f g} \int \exp \left\{i\left(k_{\mathrm{i}}-k_{m}\right) z^{\prime}\right\} V_{m \mathrm{O}}\left(\rho_{\mathrm{O}}, z^{\prime}\right) \\
& \cdot \exp \left\{i \frac{k_{m}}{2 g}\left(\rho_{\mathrm{L}}-\rho_{\mathrm{O}}\right)^{2}\right\} . \\
& \cdot \exp \left\{-i \frac{k_{m}}{2 f} \rho_{\mathrm{L}}^{2}\right\} \cdot \exp \left\{i \frac{k_{m}}{2 f}\left(\rho_{\mathrm{A}}-\rho_{\mathrm{L}}\right)^{2}\right\} \mathrm{d}^{2} \rho_{\mathrm{O}} \mathrm{d} z^{\prime} \mathrm{d}^{2} \rho_{\mathrm{L}}= \\
& =-\frac{m_{0}}{2 \pi \hbar^{2}} \frac{\exp \left\{i k_{m}(f+g)\right\}}{f} \exp \left\{-i \frac{k_{m}}{2} \frac{(g-f)}{f^{2}} \rho_{\mathrm{A}}^{2}\right\} \\
& \cdot \int \exp \left\{i\left(k_{\mathrm{i}}-k_{m}\right) z_{\mathrm{O}}\right\} V_{m 0}\left(\rho_{\mathrm{O}}, z_{\mathrm{O}}\right) \exp \left\{-i k_{m} \rho_{\mathrm{O}} \rho_{\mathrm{A}} / f\right\} \mathrm{d}^{2} \rho_{\mathrm{O}} \mathrm{d} z^{\prime} .
\end{aligned}
$$


In the last step we have performed the integration over the plane $z_{\mathrm{L}}$. Using the scattering amplitude

$$
f_{m 0}(\mathbf{K})=-\frac{m_{0}}{2 \pi \hbar^{2}} \int V_{m 0}\left(\mathbf{r}_{\mathrm{O}}\right) \exp \left\{i \mathbf{K} \mathbf{r}_{\mathrm{O}}\right\} \mathbf{d}^{2} \mathbf{r}_{\mathrm{O}}
$$

where

$\mathbf{K}=\mathbf{k}_{\mathbf{i}}-\mathbf{k}_{m}$

and

$\mathbf{k}_{m}=k_{m} \mathbf{e}_{z}+k_{m} \boldsymbol{\theta}$

we eventually obtain

$$
\varphi_{m \mathrm{~A}}\left(\rho_{\mathrm{A}}\right)=\frac{\exp \left\{i k_{m}(f+g)\right\}}{f} \exp \left\{-i \frac{k_{m}}{2} \frac{(g-f)}{f^{2}} \rho_{\mathrm{A}}^{2}\right\} f_{m 0}(\mathbf{K})
$$

Essentially, equation (39) states that the wave function in the back focal plane is proportional to the scattering amplitude. The intensity distribution is thus proportional to the differential cross section. For elastic scattering this distribution represents the diffraction pattern.

To determine the wave function in the image plane, we have to multiply $\varphi_{m A}\left(\rho_{A}\right)$ by the transmission function $T_{\mathrm{A}}\left(\rho_{\mathrm{A}}\right)$ and then compute the propagation into the image plane, thus obtaining

$$
\begin{aligned}
\varphi_{m \mathrm{~B}}\left(\rho_{\mathrm{B}}\right) & =\frac{\exp \left\{i k_{m}(b-f)\right\}}{i \lambda_{m}(b-f)} \\
& \int \varphi_{m \mathrm{~A}}\left(\rho_{\mathrm{A}}\right) T_{\mathrm{A}}\left(\boldsymbol{\rho}_{\mathrm{A}}\right) \exp \left\{i \frac{k_{m}}{2(b-f)}\left(\rho_{\mathrm{B}}-\rho_{\mathrm{A}}\right)^{2}\right\} \mathrm{d}^{2} \rho_{\mathrm{A}}= \\
& =\frac{f \cdot \exp \left\{i k_{m}(b+g)\right\}}{i \lambda_{m}(b-f)} \exp \left\{i \frac{k_{m} \rho_{\mathrm{B}}^{2}}{2(b-f)}\right\} \\
& \int f_{m 0}(\boldsymbol{\theta}) A(\boldsymbol{\theta}) \exp \{-i \gamma(\boldsymbol{\theta})\} \exp \left\{-i \frac{k_{m} f}{(b-f)} \rho_{\mathrm{B}} \boldsymbol{\theta}\right\} \mathrm{d}^{2} \boldsymbol{\theta}
\end{aligned}
$$

Here we have used $\theta=\rho_{\mathrm{A}} / f$ and the relation

$$
b-f=\frac{f^{2}}{g-f}
$$


which follows directly from the thin lens equation

$$
\frac{1}{f}=\frac{1}{b}+\frac{1}{g} .
$$

We now determine the current density $j_{\mathrm{B}}\left(\rho_{\mathrm{B}}\right)$ perpendicular to the image plane. To be independant of magnification and image inversion, we refer the current density back to the object plane. Defining $\rho=-\rho_{\mathrm{B}} \frac{g}{b}$ and scaling the resulting current density to the current density on the object, we obtain

$$
j_{m 0}(\boldsymbol{\rho})=j_{0} \frac{k_{m}}{k_{\mathrm{i}}} \frac{1}{\lambda_{m}^{2}}\left|\int f_{m 0}(\boldsymbol{\theta}) A(\boldsymbol{\theta}) \exp \{-i \gamma(\boldsymbol{\theta}, \delta E)\} \exp \left\{i k_{m} \boldsymbol{\theta} \boldsymbol{\rho}\right\} \mathrm{d}^{2} \boldsymbol{\theta}\right|^{2}
$$

This result is equivalent to equation (43) in [30] , where the sum over the products of scattering amplitudes $\sum_{m} f_{0 m}\left(\theta^{\prime}\right) f_{0 m}^{*}(\theta)$ has been expressed by the mixed dynamic form factor.

\section{References}

[1] Castaing R. and Henry L., C. R. Acad. Sci. Paris 255 (1962) 76.

[2] Henry L., Duval P. and Hoan N., C. R. Acad. Sci. Paris 269 (1969) 955.

[3] DuVAl P. and HENRY L., J. Appl. Cryst. 6 (1973) 113.

[4] CASTAING R., Physical Aspects of Electron Microscopy and Microbeam Analysis, B. Siegel and D. Beaman Eds. (Wiley, New York, 1975) p. 287.

[5] Duval P., HénOC P. and HeNRY L., C. R. Acad. Sci. Paris 271 (1970) 1108.

[6] Duval P. and Henry L., C. R. Acad. Sci. Paris 274 (1972) 684.

[7] Castaing R., El Hili A., and Henry L., C. R. Acad. Sci. Paris 262 (1966) 169;

Castaing R., El Hili A., and Henry L., C. R. Acad. Sci. Paris 262 (1966) 1051.

[8] Castaing R., Zeitschr. Angew. Phys. 27, (1969) 171.

[9] CAstaing R. and Henry L., C. R. Acad. Sci. Paris 259 (1964) 1702.

[10] MORY C. and COLlieX C., Philos. Mag. 33 (1976) 97.

[11] HÉNOC P. and HENRY L., J. Phys. Colloq. France 31 (1971) C1-55.

[12] RUTHEMANN G., Naturwiss. 30 (1942) 145.

[13] Ruthemann G., Ann. Phys. 2 (1948) $113+135$.

[14] CollieX C. and Jouffrey B., C. R. Acad. Sci. Paris 270 (1970) 673.

[15] OTTENSMEYER F.P. and ANDREW J.W., J. Ultrastruct. Res. 72 (1980) 336;

SHumaN H., ChANG C.F. and SOMLYO A.P., Ultramicroscopy 19 (1986) 121.

[16] KRAHL D., Proc. Xth Int. Congr. Electr. Micr., Vol. I (Hamburg, 1982) 173;

KRAHL D., Mat.-wiss. u. Werkstofftech. 21 (1990) 84.

[17] RUHLE M. et al, Proc. 49th EMSA Meeting (1991) p. 706.

[18] ReImer L., Adv. Electr. Electr. Phys. 81 (1991) 43.

[19] SENOUSSI S., Thèse 3ème cycle, Orsay (1971).

[20] ZaNChI G., PereZ J.P., and SeVely J., Optik 43 (1975) 195.

[21] ROSE H. and Plies E., Optik 40 (1974) 336;

ROSE H., Optik 51 (1978) 15;

LANIO S., ROSE H. and KRAHL D., Optik 73 (1986) 56.

[22] LANIO S., Optik 73 (1986) 99.

[23] KrahL D., PÄTZOLd H. and Swoboda M., Proc. XIIth Int. Congr. on Electron Microsc. (Seattle, 1990) p. 60.

[24] MAYER J., SPENCE J.C.H. and MöBUS G., Proc. 49th EMSA Meeting (1991) p. 786.

[25] SPENCE J.C.H. and MAYER J., Proc. 49th EMSA Meeting (1991) p. 616.

[26] BORn M., Ann. Phys. 38 (1926) 803. 
[27] SOMMERFELD A., Atombau und Spektrallinien (Vieweg, Braunschweig, 1951).

[28] MOTT N.F. and MASSEY H.S.W., The Theory of Atomic Collisions (Clarendon, Oxford, 1950).

[29] WU T. and OHMURA T., Quantum Theory of Scattering (Prentice-Hall, Englewood Cliffs, 1962).

[30] KOHL H. and ROSE H., Adv. Electr. Electr. Phys. 65 (1985) 173.

[31] SCHORSCH P., Diplomarbeit, TH Darmstadt (1991).

[32] SCHERZER O., J. Appl. Phys. 20 (1949) 20.

[33] MORY C., KOHL H., TENCÉ M. and COLLIEX C., Utramicroscopy 37 (1991) 191.

[34] KRIVANEK O.L., MORY C., TENCÉ M. and Colliex C., Microsc. Microanal. Microstruct. 2 (1991) 257.

[35] Castaing R., Proc. 4ème Congrès de Chimie Analytique, in press.

[36] GoODMAN J.W., Introduction to Fourier Optics (McGraw-Hill, New-York, 1968). 